\title{
Numerical Modelling of Industrial Induction
}

\author{
A. Bermúdez ${ }^{1}$, D. Gómez ${ }^{1}$, M.C. Muñiz ${ }^{1}$, P. Salgado ${ }^{1}$ and R. Vázquez ${ }^{2}$ \\ ${ }^{1}$ Departamento de Matemática Aplicada \\ Universidade de Santiago de Compostela, 15782 Santiago de Compostela \\ ${ }^{2}$ Istituto di Matematica Applicata del CNR, via Ferrata 1, 27100, Pavia \\ ${ }^{1}$ Spain \\ ${ }^{2}$ Italy
}

\section{Introduction}

Induction heating is a physical process extensively used in the metallurgical industry for different applications involving metal melting. The main components of an induction heating system are an induction coil connected to a power-supply providing an alternating electric current and a conductive workpiece to be heated, placed inside the coil. The alternating current traversing the coil generates eddy currents in the workpiece and by means of ohmic losses the workpiece is heated (see Fig. 1).

The design of an induction heating system mainly depends on its application. In this chapter we are interested in modelling the behavior of a coreless induction furnace like those used for melting and stirring. A simple sketch of this furnace is presented in Fig. 2(a). It consists of a helical copper coil and a workpiece formed by the crucible and the load within, which is the material to melt. The crucible is a cylindrical vessel made of a refractory material with higher temperature resistance than the substances it is designed to hold in. The coil, which is water-cooled to avoid overheating, is usually enclosed into a refractory material for safety reasons. Alternating current passing through the coil induces a rapidly oscillating magnetic field which generates eddy currents in the workpiece. These induced currents heat the load. When the load melts, the electromagnetic field produced by the coil interacts with
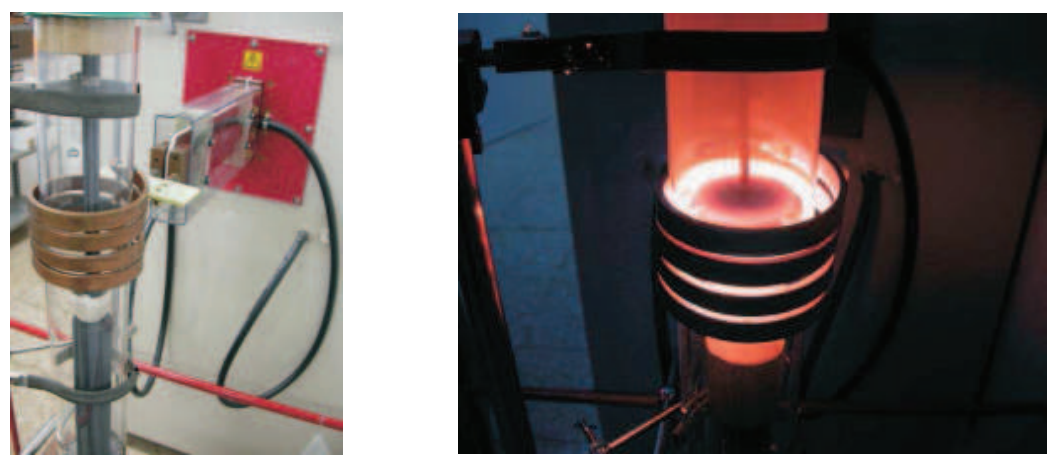

Fig. 1. Induction heating furnace off (left) and on (right). Photographs courtesy of Mr. Víctor Valcárcel, Instituto de Cerámica, Universidade de Santiago de Compostela. 

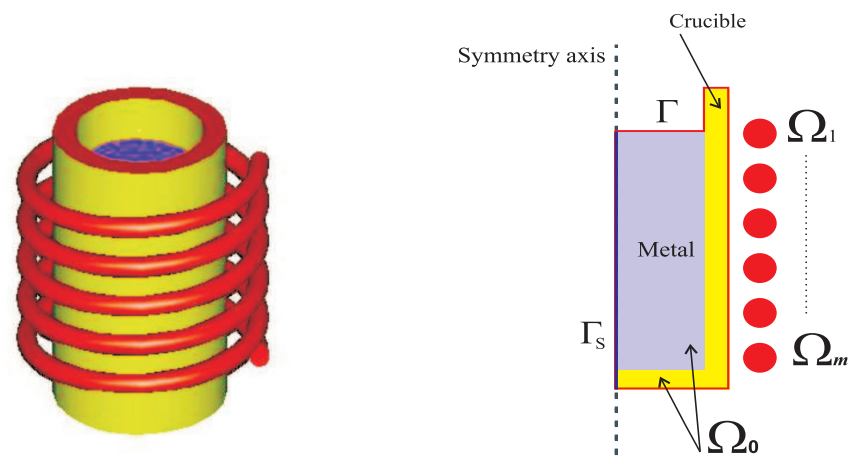

Fig. 2. Sketch of the induction furnace (a) and radial section of inductors and workpiece (b).

the electromagnetic field produced by the induced current. The resulted force causes a stirring effect helping to homogenize the melt composition and its temperature.

It is well known that the high frequencies used in induction heating applications gives rise to a phenomenon called skin effect. This skin effect forces the alternating current to flow in a thin layer close to the surface of the workpiece, at an average depth called the skin depth. Thus, the ohmic losses are concentrated in the external part of the workpiece in such a way that the higher the frequency the thinner the skin depth. It is crucial to control the distribution of these ohmic losses, since they could cause very high temperatures in the crucible thus reducing its lifetime. Moreover, the frequency and intensity of the alternating current also affect the stirring of the molten bath. Since stirring mainly determines some of the properties of the final product, it is convenient to accurately know the influence of these parameters to achieve the desired result.

Our goal is to understand the influence on the furnace performance of certain geometrical parameters such as the crucible thickness, its distance to the coil, the number of turns of the coil, or physical parameters such as the thermal and electrical conductivity of the refractory materials. In the last years, numerical simulation reveals as an important tool for this purpose, since it allows to introduce changes in the above parameters in silico, thus avoiding long and costly trial-error procedures in plant.

The overall process is very complex and involves different physical phenomena: electromagnetic, thermal with phase change and hydrodynamic in the liquid region; all of them are coupled and it is essential to consider a suitable mathematical model to achieve a realistic simulation.

Many papers have been published concerning the numerical simulation of induction heating devices, from some pioneering articles published in the early eighties (see, for instance, (Lavers, 1983) and references therein) to more recent works dealing with different coupled problems, such as the thermoelectrical problem appearing in induction heating (Chaboudez et al., 1997; Rappaz \& Swierkosz, 1996), the magnetohydrodynamic problem related to induction stirring (Natarajan \&El-Kaddah, 1999) and also a thermal-magneto-hydrodynamic problem (Henneberger \& Obrecht, 1994; Katsumura et al., 1996) but not fully coupled because material properties are not supposed to be dependent on temperature. Some other related works include mechanical effects in the workpiece (Bay et al., 2003; Hömberg, 2004). A more extensive bibliographic review can be found in (Lavers, 2008). 
The aim of this chapter is to deal with the coupled thermo-magneto-hydrodynamic simulation of an induction heating furnace like the one described above. It is a survey of previous works by the authors (Bermúdez et al., 2007;b; 2009; Vázquez, 2008). Section 2 is devoted to describe the mathematical model to compute the electromagnetic field and the temperature in the furnace, and the velocity field in the molten region; the coupled mathematical model is a system of partial differential equations with several non-linearities. In Section 3 a weak formulation of the electromagnetic model, defined in an axisymmetric setting, is derived. In Section 4 we present the time-discretization of the thermal and hydrodynamic models and the corresponding weak formulations. In Section 5 we describe the spatial discretization and the algorithms used to deal with the coupling formulation and the non-linearities. Finally, in Section 6, several numerical results are given for an industrial heating furnace designed for melting.

\section{Mathematical model for the induction furnace}

In this section we introduce the mathematical model to study the thermo-magneto-hydro dynamic behavior of an induction furnace.

\subsection{The electromagnetic model}

In order to model the electric current traversing the coil and the eddy currents induced in the workpiece, we introduce an electromagnetic model which is obtained from Maxwell equations. We state below a three-dimensional model defined in the whole space $\mathbb{R}^{3}$ and deduce an axisymmetric formulation in Section 3. Since the full description from the 3D model to the axisymmetric one involves a lot of technical steps and it has been done in (Bermúdez et al., 2009), we only give here a brief description and refer the reader to the quoted paper for further details.

We begin by introducing some assumptions and notations related with the geometry that will be used in the sequel. Since the material enclosing the coil is not only a good refractory but also a good electrical insulator, the induction process in the workpiece is almost unaffected by the presence of this material. Thus, in the electromagnetic model, it will be treated as air. For simplicity, we do not consider the water refrigerating the coil.

In order to state the problem in an axisymmetric setting, the induction coil has to be replaced by $m$ rings having toroidal geometry. Let $\Omega_{0}$ be the radial section of the workpiece and $\Omega_{1}, \Omega_{2}, \ldots, \Omega_{m}$ be the radial sections of the turns of the coil, which are assumed to be simply connected (see Fig. 2(b)). Moreover, let us denote by $\Omega$ the radial section of the set of conductors, i.e., inductors and conducting materials in the workpiece, given by,

$$
\Omega=\bigcup_{k=0}^{m} \Omega_{k},
$$

and $\Omega^{\mathrm{C}}=\mathbb{R}^{2} \backslash \bar{\Omega}$. In particular, the refractory layer enclosing the coil is part of $\Omega^{\mathrm{C}}$.

Let $\Delta \subset \mathbb{R}^{3}$ be the bounded open set generated by the rotation about the $z$-axis of $\Omega$ and $\Delta^{\mathrm{c}}$ the complementary set of $\bar{\Delta}$ in $\mathbb{R}^{3}$. Notice that $\Delta^{\mathrm{c}}$ is an unbounded set corresponding to the air surrounding the whole device. Analogously, we denote by $\Delta_{k}, k=0, \ldots, m$ the subset of $\mathbb{R}^{3}$ generated by the rotation of $\Omega_{k}, k=0, \ldots, m$, respectively, around the $z$-axis (see Fig. 3). For simplicity, we will assume that $\Delta_{0}$ is simply connected and that $\Delta_{k}, k=1, \ldots, m$ is a solid torus, in the sense that one single cutting surface $\Omega_{k}$ is enough for the set $\Delta_{k} \backslash \Omega_{k}$ to be simply-connected (see Fig. 3). 

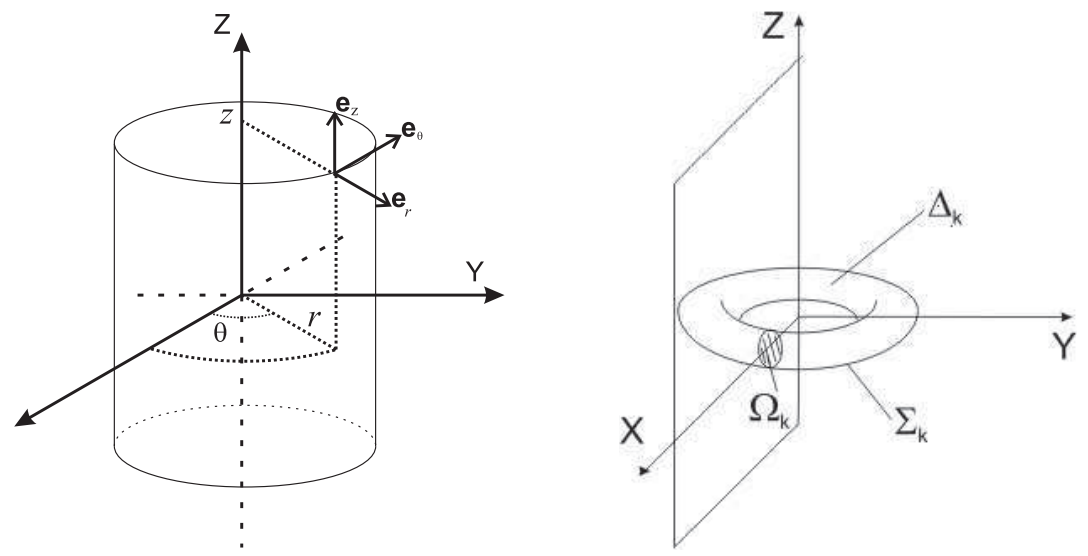

Fig. 3. Cylindrical coordinate system (a) and sketch of a toroidal turn $\Delta_{k}$ (b).

We denote by $\Sigma$ the boundary of $\Delta$ and by $\Gamma$ its intersection with the half-plane $\left\{(r, z) \in \mathbb{R}^{2} ; r>\right.$ $0\}$. We notice that $\Sigma=\cup_{k=0}^{m} \Sigma_{k}$, where $\Sigma_{k}$ denotes the boundary of $\Delta_{k}$. Moreover, we assume that the boundary of $\Omega$ is the union of $\Gamma$ and $\Gamma_{\mathrm{s}}$, the latter being a subset of the symmetry axis (see Fig. 2(b)).

The induction furnace works with alternating current so we can consider that all fields vary harmonically with time in the form:

$$
\mathcal{F}(\mathbf{x}, t)=\operatorname{Re}\left[\mathrm{e}^{\mathrm{i} \omega t} \mathbf{F}(\mathbf{x})\right],
$$

where $t$ is time, $\mathbf{x} \in \mathbb{R}^{3}$ is the spatial variable, $i$ is the imaginary unit, $\mathbf{F}(\mathbf{x})$ is the complex amplitude of field $\mathcal{F}$ and $\omega$ is the angular frequency, $\omega=2 \pi f, f$ being the frequency of the alternating current.

For low and moderate frequencies, displacement currents can be neglected. Then, Maxwell's equations can be reduced to the so-called eddy current model (see (Bossavit, 1998)):

$$
\begin{aligned}
\operatorname{curl} \mathbf{H} & =\mathbf{J} \text { in } \mathbb{R}^{3}, \\
\mathrm{i} \omega \mathbf{B}+\operatorname{curl} \mathbf{E} & =\mathbf{0} \text { in } \mathbb{R}^{3}, \\
\operatorname{div} \mathbf{B} & =0 \text { in } \mathbb{R}^{3},
\end{aligned}
$$

where $\mathbf{H}, \mathbf{J}, \mathbf{B}$ and $\mathbf{E}$ are the complex amplitudes associated with the magnetic field, the current density, the magnetic induction and the electric field, respectively.

System (2)-(4) needs to be completed with the following constitutive relations:

$$
\begin{aligned}
& \mathbf{B}=\mu \mathbf{H} \quad \text { in } \mathbb{R}^{3}, \\
& \mathbf{J}= \begin{cases}\sigma \mathbf{E} & \text { in } \Delta, \\
\mathbf{0} & \text { in } \Delta^{\mathrm{c}},\end{cases}
\end{aligned}
$$

where $\mu$ is the magnetic permeability -which is supposed to be independent of $\mathbf{H}-$ and $\sigma$ is the electric conductivity. Both are assumed to be bounded from below by a positive constant and dependent on both the spatial variable and the temperature $T$, i.e., $\mu=\mu(\mathbf{x}, T)$ and $\sigma=$ $\sigma(\mathbf{x}, T)$. Nevertheless, for the sake of simplicity, we drop this dependency in the notation until forthcoming sections. 
Notice that we have neglected the fluid motion in the Ohm's law (6), because a dimensionless analysis shows that it is negligible in comparison with the other terms for this kind of applications.

Since the previous equations hold in $\mathbb{R}^{3}$, we also require for the fields a certain behavior at infinity. More specifically, we have,

$$
\begin{aligned}
\mathbf{E}(\mathbf{x}) & =O\left(|\mathbf{x}|^{-1}\right), & & \text { uniformly for }|\mathbf{x}| \rightarrow \infty, \\
\mathbf{H}(\mathbf{x}) & =O\left(|\mathbf{x}|^{-1}\right), & & \text { uniformly for }|\mathbf{x}| \rightarrow \infty .
\end{aligned}
$$

Model (2)-(8) must be completed with some source data related to the energizing device. In particular, we would like to impose the current intensities $\mathbf{I}=\left(I_{1}, \ldots, I_{m}\right)$ crossing each transversal section of the inductor, i.e.,

$$
\int_{\Omega_{k}} \mathbf{J} \cdot v=I_{k}, k=1, \ldots, m,
$$

where $v$ denotes a unit normal vector to the section $\Omega_{k}$.

Remark 21 Imposing these constraints is not trivial at all and requires to relax in some sense equation (3). We refer the reader to Remarks 2.1 and 2.2 of (Bermúdez et al., 2009). To this respect, we also cite the paper (Alonso et al., 2008) where the authors give a systematic analysis to solve eddy current problems driven by voltage or current intensity in the harmonic regime and in bounded domains.

To solve the eddy current problem in an axisymmetric setting, we are going to propose a formulation based on the magnetic vector potential. To do that, we also need to introduce a suitable scalar potential. Firstly, equation (4) allows us to affirm that there exists a magnetic vector potential A defined in $\mathbb{R}^{3}$ such that,

$$
\mathbf{B}=\operatorname{curl} \mathbf{A},
$$

and from equation (3), we obtain

$$
\mathrm{i} \omega \operatorname{curl} \mathbf{A}+\operatorname{curl} \mathrm{E}=\mathbf{0} \text { in } \Delta .
$$

Taking into account the form of the kernel of the curl operator in each connected component of the conductor, we can say that (see, for instance (Amrouche et al., 1998))

$$
\mathrm{i} \omega \mathbf{A}+\mathbf{E}=-\mathbf{v} \text { in } \Delta,
$$

where

$$
\mathbf{v}=\operatorname{grad} \tilde{U},
$$

and $\widetilde{U}$ is a scalar potential having a constant jump through each $\Omega_{k}$, for $k=1, \cdots, m$. We have denoted by grad the gradient operator in the space $H^{1}\left(\Delta \backslash \cup_{k=1}^{m} \Omega_{k}\right)$. As we will show below, this representation allows us to impose the sources in the closed circuits $\Delta_{k}$ (see also Section 5.2 of (Hiptmair \& Sterz, 2005)).

For $k=1, \ldots, m$, let us denote by $\eta_{k}$ the solution in $\mathrm{H}^{1}\left(\Delta_{k} \backslash \Omega_{k}\right)$, unique up to a constant, of the following weak problem:

$$
\begin{aligned}
& \int_{\Delta_{k} \backslash \Omega_{k}} \sigma \operatorname{grad} \eta_{k} \cdot \operatorname{grad} \xi=0, \quad \forall \xi \in \mathrm{H}^{1}\left(\Delta_{k}\right), \\
& {\left[\eta_{k}\right]_{\Omega_{k}}=1}
\end{aligned}
$$


where $\left[\eta_{k}\right]_{\Omega_{k}}$ denotes the jump of $\eta_{k}$ through $\Omega_{k}$ along $v$.

By using functions $\eta_{k}, k=1, \cdots, m$, the scalar potential $\widetilde{U}$ can be written as (see again (Amrouche et al., 1998)),

$$
\widetilde{U}=\Phi+\sum_{k=1}^{m} V_{k} \eta_{k}
$$

with $\Phi \in \mathrm{H}^{1}(\Delta)$ and $V_{k}, k=1, \ldots, m$ being some complex numbers. From the definition of $\eta_{k}$ we deduce that $V_{k}$ is the constant jump of $\widetilde{U}$ through each surface $\Omega_{k}, k=1, \cdots, m$. From a physical point of view, these complex numbers $V_{k}$ can be interpreted as voltage drops (Hiptmair \& Sterz, 2005).

Then, taking into account that $\mathbf{H}=\mu^{-1}$ curl A and equation (2) we obtain

$$
\mathrm{i} \omega \sigma \mathbf{A}+\operatorname{curl}\left(\frac{1}{\mu} \operatorname{curl} \mathbf{A}\right)=-\sigma \mathbf{v}=-\sigma\left(\operatorname{grad} \Phi+\sum_{k=1}^{m} V_{k} \operatorname{grad} \eta_{k}\right) .
$$

Notice, however, that the vector potential $\mathbf{A}$ is not unique because it can be altered by any gradient. Thus, in order to get uniqueness we need to impose some gauge conditions. In the conductor region $\Delta$, we set $\operatorname{div}(\sigma \mathbf{A})=0$ and the boundary condition $\sigma \mathbf{A} \cdot \mathbf{n}=0$ on $\Sigma$, where $\mathbf{n}$ is a unit normal vector to $\Sigma$ outward from $\Delta$.

From these gauge conditions, we can easily deduce that $\operatorname{grad} \Phi=\mathbf{0}$. Consequently,

$$
\mathbf{v}=\sum_{k=1}^{m} V_{k} \mathbf{g r a d} \eta_{k}
$$

and equation (15) can be written as

$$
\mathrm{i} \omega \sigma \mathbf{A}+\operatorname{curl}\left(\frac{1}{\mu} \operatorname{curl} \mathbf{A}\right)=-\sigma \mathbf{v}=-\sigma \sum_{k=1}^{m} V_{k} \mathbf{g r a d} \eta_{k} \text { in } \Delta .
$$

Notice that, in particular, the electric field in $\Delta$ is given by

$$
\mathbf{E}=-\mathrm{i} \omega \mathbf{A}-\sum_{k=1}^{m} V_{k} \mathbf{g} \widetilde{\mathbf{r a d}} \eta_{k}
$$

On the other hand, in the air region, we impose the gauge conditions

$$
\operatorname{div} \mathbf{A}=0 \text { in } \Delta^{\mathrm{c}} \quad \text { and } \quad \int_{\Sigma_{j}} \mathbf{A} \cdot \mathbf{n}=0, \quad j=0, \ldots, m
$$

In Section 3 we will obtain a weak formulation from equations (16), (18) and the imposition of the current intensities (9) in the axisymmetric setting.

\subsection{The thermal model}

The electromagnetic model must be coupled with the heat equation to study the thermal effects of the electromagnetic fields in the workpiece. We will describe the equations of the model in an axisymmetric setting, paying attention to the terms coupling the thermal problem with the electromagnetic one. 


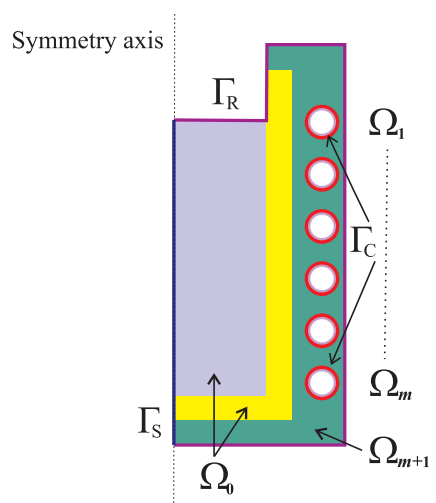

Fig. 4. Computational domain for the thermal problem

The computational domain for the thermal model is the radial section $\Omega_{\mathrm{T}}$ of the whole furnace, that is,

$$
\Omega_{\mathrm{T}}:=\Omega_{0} \cup \Omega_{1} \cup \cdots \cup \Omega_{m} \cup \Omega_{m+1},
$$

where $\Omega_{m+1}$ denotes the radial section of the dielectric parts of the furnace (see Fig. 4). We notice that we now take into account that the coil is water-cooled and replace each turn by a hollow torus. An appropriated boundary condition will be considered on the inside boundary.

Remark 22 For simplicity, in Section 2.1 the coil was replaced by rings with toroidal geometry, i.e., by solid tori. Since we are now considering a refrigeration tube along the coil, it will be replaced by hollow tori. We refer the reader to Remark 4.4 of (Vázquez, 2008) to see that, under axisymmetric assumptions, the electromagnetic model does not change in this topology.

Since the metal is introduced in solid state and then melted, we use the transient heat transfer equation with change of phase, written in terms of the enthalpy. Furthermore, since the molten metal is subject to electromagnetic and buoyancy forces, we also need to consider convective heat transfer. Let us suppose that we already know the velocity field $\mathbf{u}$ which is null in the solid part of the workpiece, and the current density J. Then, the equation for energy conservation is

$$
\dot{e}-\operatorname{div}(k(\mathbf{x}, T) \operatorname{grad} T)=\frac{|\mathbf{J}|^{2}}{2 \sigma} \text { in } \Omega_{\mathrm{T}}
$$

where $T$ is the temperature, $k$ is the temperature-dependent thermal conductivity and $\dot{e}$ is the material derivative of the enthalpy density. It is given by

$$
\dot{e}=\frac{\partial e}{\partial t}+\mathbf{u} \cdot \operatorname{grad} e .
$$

The source term on the right-hand side of (19) represents the heat released due to the Joule effect; it is obtained by solving the electromagnetic model introduced in the previous section.

Remark 23 Notice that the time scale for the variation of the electromagnetic field is much smaller than the one for the variation of the temperature. Indeed, the physical parameters used in a typical industrial situation give a time scale for temperature of the order of 60 seconds while for the 
electromagnetic problem it is of the order of $10^{-5}$ seconds. Thus, we may compute the electromagnetic field in the frequency domain using the eddy current model and then the heat source due to Joule effect is determined by taking the mean value on a cycle, namely,

$$
\frac{\omega}{2 \pi} \int_{0}^{2 \pi / \omega} \mathcal{J}(\mathbf{x}, t) \cdot \mathcal{E}(\mathbf{x}, t) d t,
$$

$\mathcal{J}$ and $\mathcal{E}$ being the current density and the electric field, respectively, written in the form of (1). It is not difficult to prove that expression (20) coincides with the right-hand side of (19).

In general, the metal in the crucible undergoes a phase change during the heating process. For this reason, the enthalpy in (19) must take into account the latent heat involved in the phase change. This can be done by introducing an enthalpy function similar to that used for Stefan problems (see (Elliot \& Ockendon, 1985)). More precisely, the enthalpy density is expressed as a multi-valued function of temperature by

$$
e(\mathbf{x}, t) \in \mathcal{H}(\mathbf{x}, T(\mathbf{x}, t)),
$$

with

$$
\mathcal{H}(\mathbf{x}, T)= \begin{cases}\Psi(\mathbf{x}, T) & T<T_{S}(\mathbf{x}), \\ {\left[\Psi\left(\mathbf{x}, T_{S}\right), \Psi\left(\mathbf{x}, T_{\mathcal{S}}\right)+L \rho\left(\mathbf{x}, T_{S}\right)\right]} & T=T_{S}(\mathbf{x}), \\ \Psi(\mathbf{x}, T)+L \rho\left(\mathbf{x}, T_{S}\right) & T>T_{S}(\mathbf{x}),\end{cases}
$$

and

$$
\Psi(\mathbf{x}, T)=\int_{0}^{T} \rho(\mathbf{x}, \zeta) c(\mathbf{x}, \zeta) d \zeta
$$

$\rho$ being the density, $c$ the specific heat and $L$ the latent heat per unit mass, i.e., the heat per unit mass necessary to achieve the change of state at temperature $T_{S}$.

Remark 24 It is to be noted that $\mathcal{H}$ is a multivalued function rather than discontinuous. Indeed, an element of volume at the solidification temperature may have any enthalpy value in the interval $\left[\Psi\left(\mathbf{x}, T_{S}\right), \Psi\left(\mathbf{x}, T_{S}\right)+\rho\left(\mathbf{x}, T_{S}\right) L\right]$.

\subsubsection{Thermal boundary conditions}

Equation (19) must be completed with suitable initial and boundary conditions. Let $\Gamma_{\mathrm{T}}$ be the boundary of $\Omega_{\mathrm{T}}$; we denote by $\Gamma_{\mathrm{S}}$ its intersection with the symmetry axis, by $\Gamma_{\mathrm{R}}$ the part of the boundary in contact with air and by $\Gamma_{C}$ the internal boundary of the coil which is in contact with the cooling water (see Fig. 4). On $\Gamma_{C}$ we consider a convection condition

$$
k(\mathbf{x}, T) \frac{\partial T}{\partial \mathbf{n}}=\alpha\left(T_{w}-T\right),
$$

$\alpha$ being the coefficient of convective heat transfer and $T_{w}$ the temperature of the cooling water. On boundary $\Gamma_{R}$ we impose the radiation-convection condition

$$
k(\mathbf{x}, T) \frac{\partial T}{\partial \mathbf{n}}=\alpha\left(T_{c}-T\right)+\gamma\left(T_{r}^{4}-T^{4}\right),
$$

where $T_{c}$ and $T_{r}$ are the external convective and radiative temperature, respectively, and $\gamma$ is the product of the emissivity by the Stefan-Boltzmann constant $\left(5.669 \mathrm{e}-8 \mathrm{~W} / \mathrm{m}^{2} \mathrm{~K}^{4}\right)$. Finally, on $\Gamma_{\mathrm{S}}$ we set the symmetry condition

$$
k(\mathbf{x}, T) \frac{\partial T}{\partial \mathbf{n}}=0 .
$$




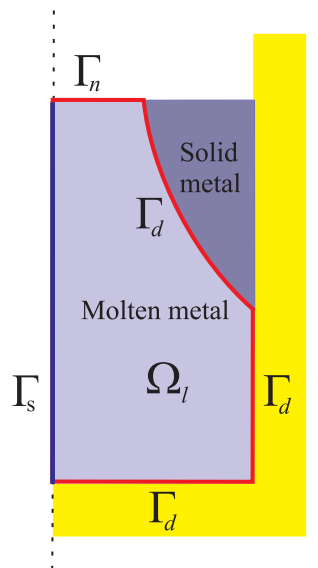

Fig. 5. Computational domain $\Omega_{l}(t)$ for the hydrodynamic problem.

\subsection{The hydrodynamic model: Boussinesq approximation}

As mentioned before, in order to achieve a realistic simulation of the overall process occurring in the furnace, convective heat transfer must be taken into account. The hydrodynamic domain is the molten region of the metal, which varies as the metal melts or solidifies, making our hydrodynamic domain time-dependent. This molten metal is subjected to both electromagnetic and buoyancy forces, the latter due to the variation of mass density with temperature.

Since the range of temperatures in the molten region is not very large, we use the Boussinesq approximation to model the fluid motion. Roughly speaking, this approximation consists on assuming that the mass density is constant in the inertial term, and that it depends linearly on the temperature in the right-hand side. Denoting by $\Omega_{l}(t)$ the radial section of the molten metal, and by $\Gamma_{\mathrm{s}}(t), \Gamma_{d}(t)$ and $\Gamma_{n}(t)$ the different parts of the boundary at time $t$ (see Fig. 5), the fluid motion is modeled by the following equations

$$
\begin{aligned}
\rho_{0}\left(\frac{\partial \mathbf{u}}{\partial t}+(\operatorname{grad} \mathbf{u}) \mathbf{u}\right)-\operatorname{div}\left(2 \eta_{0} D(\mathbf{u})\right)+\operatorname{grad} p & =-\rho_{0} \beta_{0}\left(T-T_{0}\right) \mathbf{g}+\mathbf{f}_{l}, \\
\operatorname{div} \mathbf{u} & =0,
\end{aligned}
$$

where $\mathbf{u}$ is the velocity field, $p$ is the (modified) pressure, and $D(\mathbf{u})$ is the strain rate tensor, namely $D(\mathbf{u})=\left(\operatorname{grad} \mathbf{u}+\operatorname{grad} \mathbf{u}^{t}\right) / 2$. Constants $\rho_{0}, \eta_{0}$ and $\beta_{0}$ denote the mass density, the dynamic viscosity and the thermal expansion coefficient, respectively, at the reference temperature $T_{0}$. The first term in the right-hand side takes into account the buoyancy forces ( $g$ denotes the gravity acceleration), whereas $\mathbf{f}_{l}$ represents the Lorentz force which is computed as

$$
\mathbf{f}_{l}=\frac{\omega}{2 \pi} \int_{0}^{2 \pi / \omega} \mathcal{J}(\mathbf{x}, t) \times \mathcal{B}(\mathbf{x}, t) d t,
$$

where $\mathcal{B}$ is the magnetic induction field written in the form (1). 
Equations (27)-(28) are completed with the following boundary and initial conditions:

$$
\begin{array}{rll}
\mathbf{u} & =\mathbf{0} & \text { on } \Gamma_{d}(t), \\
S \mathbf{n} & =\mathbf{0} & \text { on } \Gamma_{n}(t), \\
S \mathbf{n} & =\mathbf{0} & \text { on } \Gamma_{\mathrm{S}}(t), \\
\mathbf{u} & =\mathbf{0} & \text { in } \Omega_{l}(0),
\end{array}
$$

where $S$ denotes the Cauchy stress tensor, $S=-p I+2 \eta_{0} D(\mathbf{u})$ and $I$ is the identity tensor.

When using the Boussinesq approximation, the thermal model is modified in such a way that the material properties in the molten region are considered at the reference temperature, that is to say,

$$
\rho_{0} c_{0}\left(\frac{\partial T}{\partial t}+\mathbf{u} \cdot \operatorname{grad} T\right)-\operatorname{div}\left(k_{0} \operatorname{grad} T\right)=\frac{|\mathbf{J}|^{2}}{2 \sigma}
$$

where $c_{0}$ and $k_{0}$ represent the specific heat and the thermal conductivity at the reference temperature, respectively. We remark that this approximation is only used in the molten region of the metal. In the rest of the domain the heat equation remains non-linear.

\subsubsection{An algebraic turbulence model: Smagorinsky's model}

To take into account the possibility of turbulent flows, it is necessary to introduce a turbulence model. The basic methodology consists in modifying equations (27), (28) and (34), in the form

$$
\begin{aligned}
\rho_{0}\left(\frac{\partial \hat{\mathbf{u}}}{\partial t}+(\operatorname{grad} \hat{\mathbf{u}}) \hat{\mathbf{u}}\right)-\operatorname{div}\left(2 \eta_{e f f} D(\hat{\mathbf{u}})\right)+\operatorname{grad} \hat{p} & =-\rho_{0} \beta_{0}\left(\hat{T}-T_{0}\right) \mathbf{g}+\mathbf{f}_{l}, \\
\operatorname{div} \hat{\mathbf{u}} & =0 \\
\rho_{0} c_{0}\left(\frac{\partial \hat{T}}{\partial t}+\hat{\mathbf{u}} \cdot \operatorname{grad} \hat{T}\right)-\operatorname{div}\left(k_{e f f} \operatorname{grad} \hat{T}\right) & =\frac{|\mathbf{J}|^{2}}{2 \sigma},
\end{aligned}
$$

where $\eta_{\text {eff }}$ and $k_{\text {eff }}$ denote the effective viscosity and thermal conductivity, respectively; namely, $\eta_{e f f}=\eta_{0}+\eta_{t}$ and $k_{e f f}=k_{0}+k_{t}, \eta_{t}$ being the turbulent viscosity and $k_{t}$ the turbulent thermal conductivity.

Notice that fields $\mathbf{u}, p$ and $T$ in (27), (28) and (34) have been replaced by their corresponding filtered fields $\hat{\mathbf{u}}, \hat{p}$ and $\hat{T}$ (see (Wilcox, 2008)). Hereafter, and for the sake of simplicity, we will eliminate the symbol ^ when referring to these variables.

For a rigorous derivation of turbulence models we refer the reader to, for instance, (Mohammadi \& Pironneau, 1994; Wilcox, 2008). The turbulence models differ essentially on the way the turbulent viscosity $\eta_{t}$ and the turbulent conductivity $k_{t}$ are computed. An efficient and easy to implement model is the one proposed by Smagorinsky, which has been considered in the present work. It belongs to the family of Large Eddy Simulation (LES) models. In this case, $\eta_{t}$ and $k_{t}$ are given by

$$
\eta_{t}=\rho_{0} C h^{2}|D(\mathbf{u})|, \quad C \cong 0.01, \quad k_{t}=c_{0} \frac{\eta_{t}}{P r_{t}},
$$

where $h(\mathbf{x})$ is the mesh size of the numerical method around point $\mathbf{x}$, and $P r_{t}$ is the turbulent Prandtl number, which is taken equal to 0.9 .

It is worth noting that more accurate turbulent models, such as the well-known $k-\epsilon$ model, can also be used. The drawback is that the computational effort to apply those models is 
much higher, since they require the solution of some additional partial differential equations in order to compute the turbulent viscosity $\eta_{t}$. However, if an accurate simulation of the fluid motion is necessary -as for instance in electromagnetic stirring- the use of one of these models might become unavoidable.

\section{Weak formulation of the electromagnetic problem in an axisymmetric setting}

In this section we present the weak formulation of the electromagnetic problem. We start by introducing some functional spaces and sets. Let $\mathcal{X}$ be the Beppo-Levi space (see (Nédélec, 2001), Section 2.5.4)

$$
\mathcal{X}=\left\{\mathbf{G}: \frac{\mathbf{G}(\mathbf{x})}{\sqrt{1+|\mathbf{x}|^{2}}} \in \mathbf{L}^{2}\left(\mathbb{R}^{3}\right), \operatorname{curl} \mathbf{G} \in \mathbf{L}^{2}\left(\mathbb{R}^{3}\right)\right\},
$$

and its subset

$$
\mathcal{Y}=\left\{\mathbf{G} \in \mathcal{X}: \operatorname{div} \mathbf{G}=0 \text { in } \Delta^{\mathrm{c}}, \int_{\Sigma_{j}} \mathbf{G} \cdot \mathbf{n}=0, j=0, \ldots, m\right\}
$$

We recall that we are interested in finding a solution of the eddy current problem satisfying the intensity conditions (9). To attain this goal, we start by writing these constraints in a weak sense.

Since current density $\mathbf{J}=\sigma \mathbf{E}$ satisfies $\operatorname{div} \mathbf{J}=0$ in $\Delta$ and $\mathbf{J} \cdot \mathbf{n}=0$ on $\Sigma$, we have for $k=1, \ldots, m$,

$$
\int_{\Delta_{k} \backslash \Omega_{k}} \mathbf{J} \cdot \operatorname{grad} \eta_{k}=-\int_{\Delta_{k} \backslash \Omega_{k}} \operatorname{div} \mathbf{J} \eta_{k}+\int_{\partial\left(\Delta_{k} \backslash \Omega_{k}\right)} \mathbf{J} \cdot \mathbf{n} \eta_{k}=\int_{\Omega_{k}}\left[\eta_{k}\right] \mathbf{J} \cdot v_{k}=I_{k},
$$

where $v_{k}$ is the unit vector normal to the radial section $\Omega_{k}$. Hence, we can impose the current intensities as follows:

$$
\sum_{k=1}^{m} \bar{W}_{k} \int_{\Delta_{k} \backslash \Omega_{k}} \sigma \mathbf{E} \cdot \mathbf{g} \widetilde{\mathbf{r a d}} \eta_{k}=\sum_{k=1}^{m} I_{k} \bar{W}_{k}, \quad \forall \mathbf{W}=\left(W_{1}, \ldots, W_{m}\right) \in \mathbb{C}^{m} .
$$

Then, taking into account (17), we obtain the following weak form of constraint (9) which is well defined for any vector function $\mathbf{A} \in \mathcal{Y}$ and vector $\mathbf{W} \in \mathbb{C}^{m}$ :

$$
-\sum_{k=1}^{m} \bar{W}_{k} \int_{\Delta_{k} \backslash \Omega_{k}} \mathrm{i} \omega \sigma \operatorname{grad} \eta_{k} \cdot \mathbf{A}-\sum_{k=1}^{m} \bar{W}_{k} \int_{\Delta_{k} \backslash \Omega_{k}} \sigma V_{k}\left|\operatorname{grad} \eta_{k}\right|^{2}=\sum_{k=1}^{m} I_{k} \bar{W}_{k} .
$$

On the other hand, multiplying equation (16) by the complex conjugate of a test function $\mathrm{G}$, denoted by $\overline{\mathbf{G}}$, integrating in $\mathbb{R}^{3}$ and using a Green's formula we can easily obtain,

$$
\mathrm{i} \omega \int_{\mathbb{R}^{3}} \sigma \mathbf{A} \cdot \overline{\mathbf{G}}+\int_{\mathbb{R}^{3}} \frac{1}{\mu} \operatorname{curl} \mathbf{A} \cdot \operatorname{curl} \overline{\mathbf{G}}+\sum_{k=1}^{m} V_{k} \int_{\Delta_{k} \backslash \Omega_{k}} \sigma \operatorname{grad} \eta_{k} \cdot \overline{\mathbf{G}}, \quad \forall \mathbf{G} \in \mathcal{Y} .
$$

Thus, we are led to solve the following mixed problem:

Problem MPI.- Given $\mathbf{I}=\left(I_{1}, \ldots, I_{m}\right) \in \mathbb{C}^{m}$, find $\mathbf{A} \in \mathcal{Y}$ and $\mathbf{V} \in \mathbb{C}^{m}$, such that

$$
\begin{array}{r}
\mathrm{i} \omega \int_{\mathbb{R}^{3}} \sigma \mathbf{A} \cdot \overline{\mathbf{G}}+\int_{\mathbb{R}^{3}} \frac{1}{\mu} \operatorname{curl} \mathbf{A} \cdot \operatorname{curl} \overline{\mathbf{G}}+\sum_{k=1}^{m} V_{k} \int_{\Delta_{k} \backslash \Omega_{k}} \sigma \mathbf{g r a d} \eta_{k} \cdot \overline{\mathbf{G}}=0, \forall \mathbf{G} \in \mathcal{Y} . \\
\sum_{k=1}^{m} \bar{W}_{k} \int_{\Delta_{k} \backslash \Omega_{k}} \sigma \operatorname{grad} \eta_{k} \cdot \mathbf{A}+\frac{1}{\mathrm{i} \omega} \sum_{k=1}^{m} \bar{W}_{k} \int_{\Delta_{k} \backslash \Omega_{k}} \sigma V_{k}\left|\operatorname{grad} \eta_{k}\right|^{2}=-\frac{1}{\mathrm{i} \omega} \sum_{k=1}^{m} I_{k} \bar{W}_{k}, \forall \mathbf{W} \in \mathbb{C}^{m} .
\end{array}
$$


Notice that the vector field $\mathbf{V}$ of voltage drops, can be interpreted as a Lagrange multiplier introduced to impose the current intensities in a weak sense. The mathematical analysis of this mixed formulation can be found in (Bermúdez et al., 2009), where the simpler formulation with the voltage drops as data is also studied.

\subsection{An axisymmetric BEM/FEM formulation of problem MPI}

Notice that the above formulation is written in the whole space $\mathbb{R}^{3}$; in (Bermúdez et al., 2007) we have approximated the problem in a bounded domain by defining approximated boundary conditions far from the conducting region and using a finite element technique. However, in this work, we consider a hybrid boundary element/finite element method (in the sequel BEM/FEM) to solve the problem in the whole space. To attain this goal, we are going to write the problem MPI in another form involving only the values of the magnetic vector potential $\mathbf{A}$ in $\Delta$ and on its boundary $\Sigma$. Notice first that the field $\mu^{-1}$ curl A, which is the intensity of the magnetic field, belongs to $\mathcal{X}$, and then its tangential trace $\mu^{-1}$ curl $\mathbf{A} \times \mathbf{n}$ is continuous across $\Sigma$. Besides

$$
\operatorname{curl}\left(\frac{1}{\mu_{0}} \operatorname{curl} \mathbf{A}\right)=\operatorname{curl} \mathbf{H}=\mathbf{0} \text { in } \Delta^{\mathrm{c}},
$$

where $\mu_{0}$ denotes the vacuum magnetic permeability. Then, by using a Green's formula in $\Delta^{c}$, we have,

$$
\begin{aligned}
& \int_{\mathbb{R}^{3}} \frac{1}{\mu} \operatorname{curl} \mathbf{A} \cdot \operatorname{curl} \overline{\mathbf{G}}=\int_{\Delta} \frac{1}{\mu} \operatorname{curl} \mathbf{A} \cdot \operatorname{curl} \overline{\mathbf{G}}+\int_{\Delta^{\mathrm{c}}} \frac{1}{\mu_{0}} \operatorname{curl} \mathbf{A} \cdot \operatorname{curl} \overline{\mathbf{G}} \\
& =\int_{\Delta} \frac{1}{\mu} \operatorname{curl} \mathbf{A} \cdot \operatorname{curl} \overline{\mathbf{G}}-\int_{\Sigma} \frac{1}{\mu_{0}} \operatorname{curl} \mathbf{A} \times \mathbf{n} \cdot \overline{\mathbf{G}}, \forall \mathbf{G} \in \mathcal{Y} .
\end{aligned}
$$

Thus, the first equation of problem MPI can be formally written as:

$$
\begin{aligned}
& \mathrm{i} \omega \int_{\Delta} \sigma \mathbf{A} \cdot \overline{\mathbf{G}}+\int_{\Delta} \frac{1}{\mu} \operatorname{curl} \mathbf{A} \cdot \operatorname{curl} \overline{\mathbf{G}}-\int_{\Sigma} \frac{1}{\mu_{0}} \operatorname{curl} \mathbf{A} \times \mathbf{n} \cdot \overline{\mathbf{G}} \\
& +\sum_{k=1}^{m} V_{k} \int_{\Delta_{k} \backslash \Omega_{k}} \sigma \operatorname{grad} \eta_{k} \cdot \overline{\mathbf{G}}=0, \forall \mathbf{G} \in \mathcal{Y} .
\end{aligned}
$$

We notice that the value of $\mu_{0}^{-1}$ curl $\mathbf{A} \times \mathbf{n}$ on $\Sigma$ can be determined by solving an exterior problem in $\Delta^{\mathrm{c}}$. Since we are interested in the numerical solution of the problem in an axisymmetric domain, we directly transform this term in the axisymmetric case.

We consider a cylindrical coordinate system $(r, \theta, z)$ with the $z$-axis coinciding with the symmetry axis of the device, (see Figure 3). Hereafter we denote by $\mathbf{e}_{r}, \mathbf{e}_{\theta}$ and $\mathbf{e}_{z}$ the local unit vectors in the corresponding coordinate directions. Now, cylindrical symmetry leads us to consider that no field depends on the angular variable $\theta$. We further assume that the current density field has non-zero component only in the tangential direction $\mathbf{e}_{\theta}$, namely

$$
\mathbf{J}=J_{\theta}(r, z) \mathbf{e}_{\theta} .
$$

We remark that, due to the assumed conditions on J, (3), (6) and (10), only the $\theta$-component of the magnetic vector potential, hereafter denoted by $A_{\theta}$, does not vanish, i.e.,

$$
\mathbf{A}=A_{\theta}(r, z) \mathbf{e}_{\theta} .
$$


Thus A automatically satisfies (18). Let $\mathbf{G}=\psi(r, z) \mathbf{e}_{\theta}$ be a test function and $\mathbf{n}=n_{r} \mathbf{e}_{r}+n_{z} \mathbf{e}_{z}$. By taking into account the expression for curl in cylindrical coordinates and the fact that

$$
\operatorname{grad} \eta_{k}=\frac{1}{2 \pi r} \mathbf{e}_{\theta}, \quad \text { in } \Delta_{k}, k=1, \ldots, m,
$$

the axisymmetric version of the problem formally writes as follows:

Given $\mathbf{I}=\left(I_{1}, \ldots, I_{m}\right) \in \mathbb{C}^{m}$, find $A_{\theta}$ and $\mathbf{V} \in \mathbb{C}^{m}$, satisfying,

$$
\begin{aligned}
& \mathrm{i} \omega \int_{\Omega} \sigma A_{\theta} \cdot \bar{\psi} r d r d z+\int_{\Omega} \frac{1}{\mu r} \frac{\partial\left(r A_{\theta}\right)}{\partial r} \frac{\partial(r \bar{\psi})}{\partial r} d r d z+\int_{\Omega} \frac{1}{\mu} \frac{\partial A_{\theta}}{\partial z} \frac{\partial \bar{\psi}}{\partial z} r d r d z \\
& -\int_{\Gamma} \frac{1}{\mu_{0}} \frac{\partial\left(r A_{\theta}\right)}{\partial \mathbf{n}} \bar{\psi} d \gamma+\frac{1}{2 \pi} \sum_{k=1}^{m} V_{k} \int_{\Omega_{k}} \sigma \bar{\psi} d r d z=0 \\
& \frac{1}{2 \pi} \sum_{k=1}^{m}\left(\int_{\Omega_{k}} \sigma A_{\theta} d r d z\right) \bar{W}_{k}+\frac{1}{4 \pi^{2} \mathrm{i} \omega} \sum_{k=1}^{m}\left(\int_{\Omega_{k}} \sigma \frac{V_{k}}{r} d r d z\right) \bar{W}_{k}=-\frac{1}{2 \pi \mathrm{i} \omega} \sum_{k=1}^{m} I_{k} \bar{W}_{k},
\end{aligned}
$$

for all test function $\psi$ and $W \in \mathbb{C}^{m}$.

On the other hand, the term $\int_{\Gamma} \mu_{0}^{-1} \partial\left(r A_{\theta}\right) / \partial \mathbf{n} \bar{\psi} d \gamma$ can be transformed by using the single-double layer potentials. We refer the reader to (Bermúdez et al., 2007b) for further details concerning this transformation and introduce the same notation of that paper, namely

$$
\begin{aligned}
A_{\theta}^{\prime} & =r A_{\theta}, \\
\lambda^{\prime}(r, z) & =\frac{\partial A_{\theta}^{\prime}}{\partial r} n_{r}+\frac{\partial A_{\theta}^{\prime}}{\partial z} n_{z} .
\end{aligned}
$$

We are led to solve the following weak problem

Problem WEPI.- Given $\mathbf{I}=\left(I_{1}, \ldots, I_{m}\right) \in \mathbb{C}^{m}$, find $A_{\theta}^{\prime}: \Omega \longrightarrow \mathbb{C}, \mathbf{V} \in \mathbb{C}^{m}$ and $\lambda^{\prime}: \Gamma \longrightarrow \mathbb{C}$ such that

$$
\begin{array}{r}
\mathrm{i} \omega \int_{\Omega} \frac{\sigma}{r} A_{\theta}^{\prime} \bar{\psi}^{\prime} d r d z+\int_{\Omega} \frac{1}{\mu r} \operatorname{grad} A_{\theta}^{\prime} \cdot \operatorname{grad} \bar{\psi}^{\prime} d r d z-\int_{\Gamma} \frac{1}{\mu r} \lambda^{\prime} \bar{\psi}^{\prime} d \gamma+\frac{1}{2 \pi} \sum_{k=1}^{m} V_{k} \int_{\Omega_{k}} \frac{\sigma}{r} \bar{\psi}^{\prime} d r d z=0, \\
\frac{1}{2 \pi} \sum_{k=1}^{m} \bar{W}_{k} \int_{\Omega_{k}} \frac{\sigma}{r} A_{\theta}^{\prime} d r d z+\frac{1}{4 \pi^{2} \mathrm{i} \omega} \sum_{k=1}^{m} \bar{W}_{k} \int_{\Omega_{k}} \sigma \frac{V_{k}}{r} d r d z=-\frac{1}{2 \pi \mathrm{i} \omega} \sum_{k=1}^{m} I_{k} \bar{W}_{k} \\
\int_{\Gamma} \frac{1}{\mu r} A_{\theta}^{\prime} \bar{\zeta} d \gamma-\int_{\Gamma} \frac{1}{\mu}\left(\mathcal{G}_{n} A_{\theta}^{\prime}\right) \bar{\zeta} d \gamma+\int_{\Gamma} \frac{1}{\mu}\left(\mathcal{G} \lambda^{\prime}\right) \bar{\zeta} d \gamma=0
\end{array}
$$

for all test functions $\psi^{\prime}, \zeta$ and $W \in \mathbb{C}^{m}$. In the previous equations, $\mathcal{G}$ and $\mathcal{G}_{n}$ denote the fundamental solution of Laplace's equation and its normal derivative, respectively, in cylindrical coordinates (see again (Bermúdez et al., 2007b)).

\section{Time discretization and weak formulation of the thermal and hydrodynamic problems}

In this section we introduce a time discretization and a weak formulation of the thermal and hydrodynamic models. Again, we exploit the cylindrical symmetry and we assume that $\mathbf{u}$ 
does not depend on $\theta$ and it has zero component in the tangential direction $\mathbf{e}_{\theta}$. In order to simplify the notation, in what follows we shall drop index $t$ for $\Omega_{l}(t)$.

To obtain a suitable discretization of the material time derivative in equations (19) and (27) we shall use the characteristics method (see, for instance, (Pironneau, 1982)).

Given a velocity field $\mathbf{u}$ we define the characteristic curve passing through point $\mathbf{x}$ at time $t$ as the solution of the following Cauchy problem:

$$
\left\{\begin{array}{l}
\frac{\mathrm{d}}{\mathrm{d} \tau} \mathbf{X}(\mathbf{x}, t ; \tau)=\mathbf{u}(\mathbf{X}(\mathbf{x}, t ; \tau), \tau) \\
\mathbf{X}(\mathbf{x}, t ; t)=\mathbf{x}
\end{array}\right.
$$

Thus $\mathbf{X}(\mathbf{x}, t ; \tau)$ is the trajectory of the material point being at position $\mathbf{x}$ at time $t$. In terms of $\mathbf{X}$, the material time derivative of $e$ is defined by

$$
\dot{e}(\mathbf{x}, t)=\frac{d}{d \tau}[e(\mathbf{X}(\mathbf{x}, t ; \tau), \tau)]_{\mid \tau=t} .
$$

Let us consider a time interval $\left[0, t_{f}\right]$ and a discretization time step $\Delta t=t_{f} / N$ to obtain a uniform partition $\Pi=\left\{t^{n}=n \Delta t, 0 \leq n \leq N\right\}$. Let $e^{n}$ and $\mathbf{u}^{n}$ be the approximations of $e$ and $\mathbf{u}$ at time $t^{n}$, respectively. We approximate the material time derivative of $e$ at time $t^{n+1}$ by

$$
\dot{e}\left(\mathbf{x}, t^{n+1}\right) \simeq \frac{e^{n+1}(\mathbf{x})-e^{n}\left(\chi^{n}(\mathbf{x})\right)}{\Delta t},
$$

where $\chi^{n}(\mathbf{x})=\mathbf{X}^{n}\left(\mathbf{x}, t^{n+1} ; t^{n}\right)$ is obtained as the solution of the following Cauchy problem

$$
\left\{\begin{array}{l}
\frac{\mathrm{d}}{\mathrm{d} \tau} \mathbf{X}^{n}\left(\mathbf{x}, t^{n+1} ; \tau\right)=\mathbf{u}^{n}\left(\mathbf{X}^{n}\left(\mathbf{x}, t^{n+1} ; \tau\right), \tau\right), \\
\mathbf{X}^{n}\left(\mathbf{x}, t^{n+1} ; t^{n+1}\right)=\mathbf{x},
\end{array}\right.
$$

at time $t^{n}$. Notice that, since $\mathbf{u}=\mathbf{0}$ in the solid region, the solution of this Cauchy problem is $\mathbf{X}^{n}\left(\mathbf{x}, t^{n+1} ; \tau\right)=\mathbf{x}$ for any $\tau$. Hence equation (50) in the solid part is equivalent to a standard time discretization without using the method of characteristics.

Analogous to (50), we consider in (35) the above two-point discretization for the material time derivative of the velocity. Thus, taking into account the cylindrical symmetry, multiplying equations (35) and (36) by suitable test functions, and integrating in the liquid domain $\Omega_{l}$ we obtain, after using the Green's formula, the following weak formulation of the semi-discretized hydrodynamic problem (the : representing the scalar product of two tensors)

Problem WHP.- For each $n=0,1, \ldots, N-1$, find functions $\mathbf{u}^{n+1}$ and $p^{n+1}$ such that $\mathbf{u}^{n+1}=0$ on $\Gamma_{d}$ and furthermore

$$
\begin{array}{r}
\frac{1}{\Delta t} \int_{\Omega_{l}} \rho_{0} \mathbf{u}^{n+1} \cdot \mathbf{w} r d r d z+\int_{\Omega_{l}} \eta_{e f f}\left(\operatorname{grad}^{n+1}: \operatorname{grad} \mathbf{w}\right) r d r d z+ \\
\int_{\Omega_{l}} \eta_{e f f}\left(\left(\operatorname{grad} \mathbf{u}^{n+1}\right)^{t}: \operatorname{grad} \mathbf{w}\right) r d r d z-\int_{\Omega_{l}} p^{n+1} \operatorname{div} \mathbf{w} r d r d z= \\
-\int_{\Omega_{l}} \rho_{0} \beta_{0}\left(T^{n}-T_{0}\right) \mathbf{g} \cdot \mathbf{w} r d r d z+\int_{\Omega_{l}} \mathbf{f}_{l}^{n+1} \cdot \mathbf{w} r d r d z+\frac{1}{\Delta t} \int_{\Omega_{l}} \rho_{0}\left(\mathbf{u}^{n} \circ \chi^{n}\right) \cdot \mathbf{w} r d r d z \\
\int_{\Omega_{l}} \operatorname{div} \mathbf{u}^{n+1} q r d r d z=0
\end{array}
$$


for all test functions $\mathbf{w}$ null on $\Gamma_{d}$, and $q$.

On the other hand, assuming cylindrical symmetry and the fact that the temperature does not depend on the angular coordinate $\theta$, we can write equation (19) in cylindrical coordinates. Then, applying the time discretization (50), multiplying by a suitable test function and using a Green's formula we obtain the following weak formulation of the semi-discretized thermal problem:

Problem WTP.- For each $n=0,1, \ldots, N-1$, find a function $T^{n+1}$ such that

$$
\begin{array}{r}
\int_{\Omega_{T}} \frac{1}{\Delta t} e^{n+1} Z r d r d z+\int_{\Omega_{T}} k_{e f f}\left(r, z, T^{n+1}\right) \operatorname{grad} T^{n+1} \cdot \operatorname{grad} Z r d r d z \\
=\int_{\Gamma_{C}} \alpha\left(T_{w}-T^{n+1}\right) Z r d \Gamma+\int_{\Gamma_{R}}\left(\alpha\left(T_{C}-T^{n+1}\right)+\gamma\left(T_{r}^{4}-\left(T^{n+1}\right)^{4}\right)\right) Z r d \Gamma \\
+\int_{\Omega_{T}} \frac{1}{\Delta t}\left(e^{n} \circ \chi^{n}\right) Z r d r d z+\int_{\Omega_{T}} \frac{1}{2 \sigma\left(r, z, T^{n+1}\right)}\left|J_{\theta}^{n+1}\right|^{2} Z r d r d z,
\end{array}
$$

for all test function $\mathrm{Z}$.

Remark 4.1 Note that, from equations (6), (17) and the expression for functions $\eta_{k}$, we can infer that

$$
\begin{aligned}
J_{\theta}=-\mathrm{i} \omega \sigma A_{\theta} & \text { in } \Omega_{0}, \\
J_{\theta}=-\mathrm{i} \omega \sigma A_{\theta}-\frac{V_{k}}{2 \pi r} & \text { in } \Omega_{k}, \quad k=1, \ldots, m, \\
J_{\theta}=0 & \text { in } \Omega_{m+1} .
\end{aligned}
$$

Hence, to determine the heat source we need to compute an approximation of field $A_{\theta}$ at time $t^{n+1}$. This is determined as the solution of the weak formulation WEPI with the physical parameters $\mu$ and $\sigma$ being evaluated at temperature $T^{n+1}$.

\section{Space discretization and iterative algorithm}

Problem WTP has been spatially discretized by a piecewise linear finite element method defined in a triangular mesh of the domain $\Omega_{\mathrm{T}}$. For the spatial discretization of problem WEPI we have used a BEM/FEM method (see (Bermúdez et al., 2007b) for further details). Finally, problem WHP has been spatially discretized by the finite element couple $P_{1}$-bubble $/ P_{1}$, which is known to satisfy the inf-sup condition (Brezzi, 1991); this last problem only takes place in the time-dependent liquid domain $\Omega_{l}(t)$, which must be computed at each time step.

It is important to notice that, at each time step, there are several terms coupling the three problems. However, since we are neglecting the velocity in Ohm's law, and the method of characteristics is used with the velocity at the previous time step, the hydrodynamic problem can be solved uncoupled from the two others. Nevertheless, the coupling between the thermal and the electromagnetic models cannot be avoided: the heat source in the thermal equation is the Joule effect and the solution of the electromagnetic problem depends on the electrical conductivity and the magnetic permeability, which may vary with temperature.

Moreover, the thermal problem WTP contains several nonlinearities:

- The thermal conductivity $k$ depends on temperature.

- The external convective temperature $T_{w}$ also varies with the heat flux of the inner boundaries of the coil. 
- The enthalpy $e$ depends on temperature and it is a multivalued function.

- The radiation boundary condition depends on $T^{4}$.

To treat the nonlinear terms we are going to introduce several iterative algorithms. The dependence of the thermal conductivity $k$ can be easily treated, just by taking in the heat equation the thermal conductivity evaluated at the temperature of the previous time step (or of the previous iteration of an outer loop). This can be done because the thermal conductivity is a smooth function. Nevertheless, as we will show in the next section, the other nonlinearities need other more sophisticated iterative algorithms to be introduced.

\subsection{Iterative algorithm for the temperature of cooling water}

As we said before, the induction coil of the furnace is water-cooled to avoid overheating; this fact is modeled by means of boundary condition (24), where the temperature of the cooling water, $T_{w}$, depends on the solution of our problem which introduces a nonlinearity in the equations.

To deal with this nonlinearity we will seek the convergence of the heat flux from the coil to the cooling water. From the solution of the thermal problem this heat flux is computed as

$$
H=2 \pi \int_{\Gamma_{C}} k \frac{\partial T}{\partial \mathbf{n}} r d \Gamma
$$

where the integral is multiplied by $2 \pi$ because $\Gamma_{C}$ is only a radial section of the boundary. Using (52) the outlet water temperature can be computed as

$$
T_{o}=T_{i}+\frac{H}{\rho_{w} c_{w} Q}
$$

with $\rho_{w}$ and $c_{w}$ the density and specific heat of water, respectively, $T_{i}$ the inlet temperature of the cooling water, and $Q$ the water flow rate.

To solve the problem, we assume that water temperature $T_{w}$ is constant along the coil which is actually a reasonable assumption, since the difference between the inlet and the outlet temperature is seldom higher than $10^{\circ} \mathrm{C}$.

The temperature of the cooling water is then computed using an iterative algorithm. Let us suppose $T_{w, j}$ is known. Then, at iteration $j+1$, we compute $T_{j+1}$ as the solution of thermal problem WTP with $T_{w}=T_{w, j}$. Then, we compute the heat flux $H$ from equation (52) and set

$$
T_{w, j+1}=T_{i}+\frac{H}{2 \rho_{w} c_{w} Q},
$$

i.e., $T_{w, j+1}$ is the mean value of the given inlet temperature and the computed outlet temperature.

It is worth noting that an implicit algorithm is needed to avoid instabilities. The iterations of the implicit method can be merged with the iterations of the thermoelectrical coupling leading to a low computational cost.

\subsection{Iterative algorithms for the enthalpy and the radiation boundary condition}

In order to solve the non-linearities due to the multi-valued character of the enthalpy and to the exterior radiation boundary condition, we propose a fixed point algorithm which is described in detail in references (Bermúdez et al., 2003; 2007; Vázquez, 2008). It is summarized here for the reader's convenience. 
At time step $(n+1)$ and for a positive $\beta$ we introduce the new function

$$
q^{n+1}=e^{n+1}-\beta T^{n+1} .
$$

Using (21) and the fact that $\mathcal{H}$ is a maximal monotone operator, we get

$$
q^{n+1}(r, z)=\mathcal{H}_{\lambda}^{\beta}\left((r, z), T^{n+1}(r, z)+\lambda q^{n+1}(r, z)\right) \text { for } 0<\lambda \leq \frac{1}{2 \beta},
$$

$\mathcal{H}_{\lambda}^{\beta}$ being the Yosida approximation of the operator $\mathcal{H}^{\beta}=\mathcal{H}-\beta \mathcal{I}$ (Bermúdez-Moreno, 1994). The same idea is introduced to deal with the nonlinearity associated to the fourth power of the boundary temperature in (25): we consider the maximal monotone operator $\mathcal{G}(T)=|T| T^{3}$, which coincides with $T^{4}$ for $T>0$, and we define the new function

$$
s^{n+1}=\mathcal{G}\left(T^{n+1}\right)-\kappa T^{n+1} .
$$

Then using $\mathcal{G}_{\delta}^{\kappa}$, the Yosida approximation of operator $\mathcal{G}^{\kappa}=\mathcal{G}-\kappa \mathcal{I}$, we get

$$
s^{n+1}(r, z)=\mathcal{G}_{\delta}^{\kappa}\left(T^{n+1}(r, z)+\delta s^{n+1}(r, z)\right) \text { for } 0<\delta \leq \frac{1}{2 \kappa} .
$$

Now, in order to solve problem $\mathbf{W T P}$, the idea is to replace $e^{n+1}$ by $q^{n+1}+\beta T^{n+1}$ and $\mathcal{G}\left(T^{n+1}\right)$ by $s^{n+1}+\kappa T^{n+1}$. Finally, to determine $T^{n+1}, q^{n+1}$ and $s^{n+1}$ we introduce an iterative process using (54) and (55).

We notice that the performance of the proposed algorithm is known to depend strongly on the choice of parameters $\beta, \lambda, \delta$ and $\kappa$. In (Vázquez, 2008) an automatic procedure is proposed to compute these parameters as functions of $(r, z)$, which accelerates the convergence of the method.

\subsection{Iterative algorithm for the whole problem}

Now we present the iterative algorithm to solve the three coupled models, along with the nonlinearities. Basically, it consists of three nested loops: the first one for the time discretization, the second one for the thermoelectrical coupling, and the third one for the Bermúdez-Moreno algorithms for the enthalpy and the radiation boundary condition presented above. As we have said before, the hydrodynamic problem is solved at each time step, uncoupled from the two others. Moreover, the nonlinearities of the thermal conductivity $k$ and the cooling water temperature $T_{w}$ are also treated by iterative algorithms and their corresponding loops are in fact merged with that of the thermoelectrical coupling. For the sake of simplicity we present in Fig. 6 a sketch of the algorithm with the three nested loops.

\subsubsection{The algorithm}

Let us suppose that the initial temperature $T^{0}$ and velocity $\mathbf{u}^{0}$ are known. From $T^{0}$ we determine the initial enthalpy $e^{0}$ and set the temperature of cooling water $T_{w}^{0}=T_{i}$, being $T_{i}$ the inlet temperature. Then, at time step $n$, with $n=1, \ldots, N$ we compute $A_{\theta}^{n}, T^{n}$ and $\mathbf{u}^{n}$ by doing the following steps:

1. If $\mathbf{u}^{n-1} \neq \mathbf{0}$, compute $\chi^{n-1}(\mathbf{x})$, the solution of (51).

2. Calculate the turbulent viscosity $\eta_{t}^{n}=\rho_{0} C h^{2}\left|D\left(\mathbf{u}^{n-1}\right)\right|$ and the turbulent thermal conductivity $k_{t}^{n}=c_{0} \eta_{t}^{n} / P r_{t}$. 


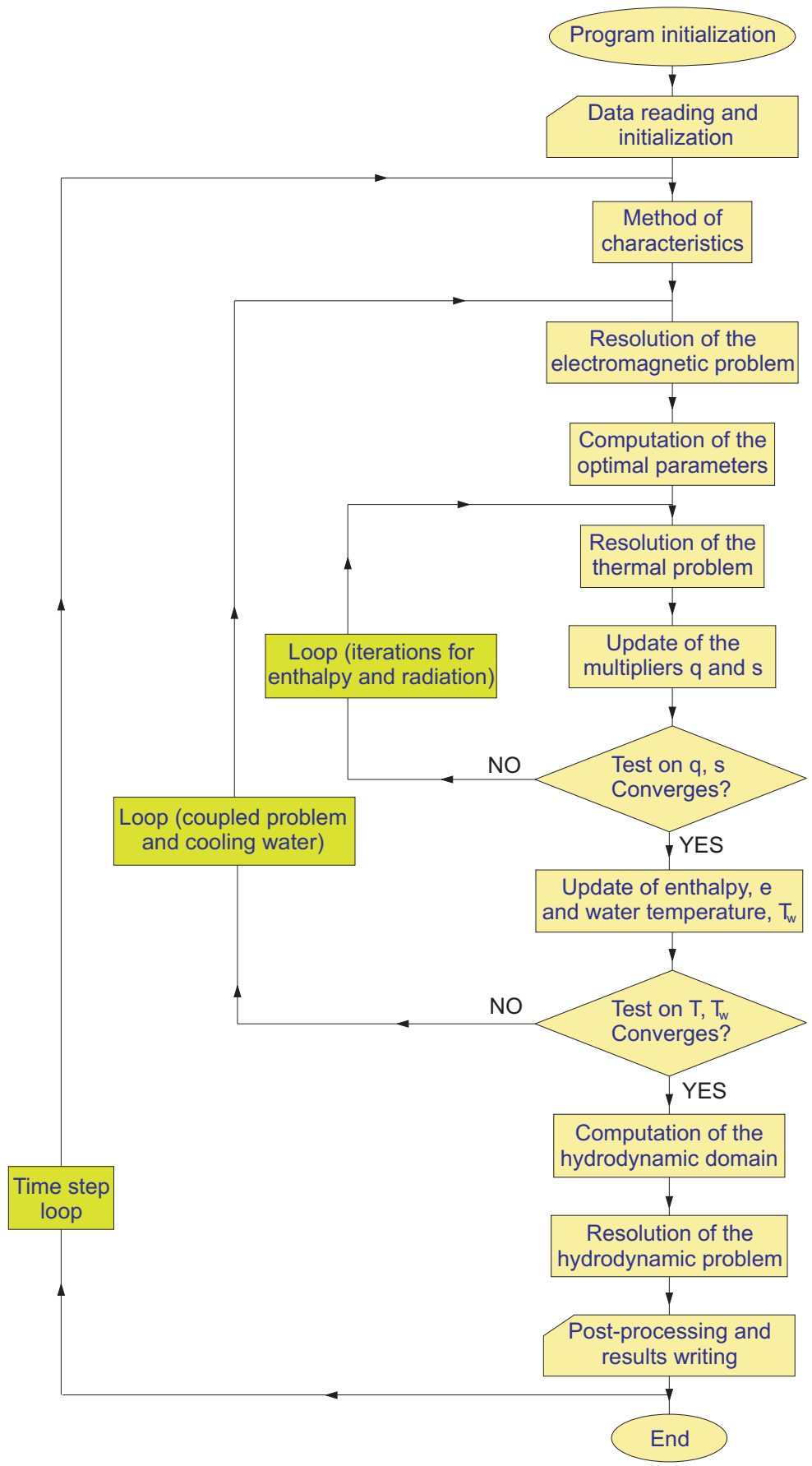

Fig. 6. Scheme of the numerical algorithm considering all the nested loops. 
3. Set $T_{0}^{n}=T^{n-1}, e_{0}^{n}=e^{n-1}$ and $T_{w, 0}^{n}=T_{w}^{n-1}$. Then compute $A_{\theta}^{n}, T^{n}, e^{n}$ and $T_{w}^{n}$ as the limit of $A_{\theta, j}^{n}, T_{j}^{n}$ and $T_{w, j}^{n}$, obtained from the following iterative procedure:

1. For $j \geq 1$ let us suppose that $T_{j-1}^{n}$ and $T_{w, j-1}^{n}$ are known. Then set $A_{\theta, j}^{n}=A_{\theta}^{\prime} / r$, with $A_{\theta^{\prime}}^{\prime}$ $\lambda^{\prime}$ and $\mathbf{V} \in \mathbb{C}^{m}$ the solution of WEPI by taking $\sigma=\sigma\left(r, z, T_{j-1}^{n}\right)$ and $\mu=\mu\left(r, z, T_{j-1}^{n}\right)$.

(b) Set $J_{\theta, j}^{n}=-\mathrm{i} \omega \sigma\left(r, z, T_{j-1}^{n}\right) A_{\theta, j}^{n}-V_{k} /(2 \pi r)$ in $\Omega_{k}, k=0, \ldots, m$ (with $V_{0}=0$ ).

(c) Determine the optimal parameters $\kappa_{j}^{n}(r, z)$ and $\beta_{j}^{n}(r, z)$ with the method described in (Vázquez, 2008).

(d) Set $T_{j, 0}^{n}=T_{j-1}^{n}$ and also

$$
\begin{aligned}
q_{j, 0}^{n} & =e_{j-1}^{n}-\beta_{j}^{n} T_{j-1}^{n}, \\
s_{j, 0}^{n} & =\left|T_{j-1}^{n}\right|\left(T_{j-1}^{n}\right)^{3}-\kappa_{j}^{n} T_{j-1}^{n} .
\end{aligned}
$$

Then compute $T_{j}^{n}, q_{j}^{n}$ and $s_{j}^{n}$ as the limit of the following iterative procedure:

1. For $k \geq 1$ let us suppose that $q_{j, k-1}^{n}$ and $s_{j, k-1}^{n}$ are known. Then compute $T_{j, k}^{n}$ as the solution of the linear system

$$
\begin{array}{r}
\int_{\Omega_{T}} \frac{1}{\Delta t} \beta_{j}^{n} T_{j, k}^{n} Z r d r d z+\int_{\Omega_{T}}\left(k\left(T_{j-1}^{n}\right)+k_{t}^{n}\right) \operatorname{grad} T_{j, k}^{n} \cdot \operatorname{grad} Z r d r d z+\int_{\Gamma_{C}} \alpha T_{j, k}^{n} Z r d \Gamma \\
+\int_{\Gamma_{R}}\left(\alpha+\gamma \kappa_{j}^{n}\right) T_{j, k}^{n} Z r d \Gamma=\int_{\Gamma_{C}} \alpha T_{w, j-1}^{n} Z r d \Gamma+\int_{\Gamma_{R}}\left(\alpha T_{\mathcal{C}}+\gamma T_{r}^{4}-\gamma s_{j, k-1}^{n}\right) Z r d \Gamma \\
+\int_{\Omega_{T}} \frac{1}{\Delta t}\left(e^{n-1} \circ \chi^{n-1}-q_{j, k-1}^{n}\right) Z r d r d z+\int_{\Omega_{T}} \frac{1}{2 \sigma\left(r, z, T_{j-1}^{n}\right)}\left|J_{\theta, j}^{n}\right|^{2} Z r d r d z \quad \forall Z \text { test function. }
\end{array}
$$

ii. Update multipliers $q_{j, k}^{n}$ and $s_{j, k}^{n}$ by means of the formulas

$$
\begin{aligned}
q_{j, k}^{n} & =\mathcal{H}_{\lambda}^{\beta}\left(T_{j, k}^{n}+\lambda q_{j, k-1}^{n}\right), \\
s_{j, k}^{n} & =\mathcal{G}_{\delta}^{\kappa}\left(T_{j, k}^{n}+\delta s_{j, k-1}^{n}\right) .
\end{aligned}
$$

(e) Update the value of the enthalpy and the value of the cooling water temperature by computing

$$
\begin{gathered}
e_{j}^{n}=q_{j}^{n}+\beta_{j}^{n} T_{j}^{n}, \\
H=2 \pi \int_{\Gamma_{C}} k \frac{\partial T_{j}^{n}}{\partial \mathbf{n}} r d \Gamma, \quad \text { and } \quad T_{w, j}^{n}=T_{i}+\frac{H}{2 \rho_{w} c_{w} Q} .
\end{gathered}
$$

4. Determine the hydrodynamic domain from the value of enthalpy $e^{n}$. 
5. Find $\mathbf{u}^{n}$ and $p^{n}$ solution of

$$
\begin{array}{r}
\frac{1}{\Delta t} \int_{\Omega_{l}} \rho_{0} \mathbf{u}^{n} \cdot \mathbf{w} r d r d z+\int_{\Omega_{l}}\left(\eta_{0}+\eta_{t}^{n}\right)\left(\operatorname{grad} \mathbf{u}^{n}: \operatorname{grad} \mathbf{w}\right) r d r d z \\
+\int_{\Omega_{l}}\left(\eta_{0}+\eta_{t}^{n}\right)\left(\left(\operatorname{grad} \mathbf{u}^{n}\right)^{t}: \operatorname{grad} \mathbf{w}\right) r d r d z-\int_{\Omega_{l}} p^{n} \operatorname{div} \mathbf{w} r d r d z= \\
-\int_{\Omega_{l}} \rho_{0} \beta_{0}\left(T^{n}-T_{0}\right) \mathbf{g} \cdot \mathbf{w} r d r d z+\int_{\Omega_{l}} \mathbf{f}_{l}\left(A_{\theta}^{n}\right) \cdot \mathbf{w} r d r d z+ \\
\frac{1}{\Delta t} \int_{\Omega_{l}} \rho_{0}\left(\mathbf{u}^{n-1} \circ \chi^{n-1}\right) \cdot \mathbf{w} r d r d z, \quad \forall \text { test function } \mathbf{w}, \\
\int_{\Omega_{l}} \operatorname{div} \mathbf{u}^{n} q=0, \quad \forall \text { test function } q .
\end{array}
$$

\section{Numerical simulation of an industrial furnace}

In this section we present some numerical results obtained in the simulation of an industrial furnace used for melting and stirring. The results have been performed with the computer Fortran code THESIF (http://www.usc.es/ thesif/) which implements the algorithm described above.

\subsection{Description of the furnace}

We briefly describe the geometry and working conditions of the furnace and refer the reader to Chapter 4 of (Vázquez, 2008) for further details.

The inductor of the furnace is a copper helical coil with 12 turns which contains a pipe inside carrying cool water for refrigeration. Inside the coil a crucible is placed, containing the metal to be melted. The crucible is surrounded by an alumina layer to avoid heat losses. The reason to take alumina for this layer is that it is not only a good refractory but also a good electrical insulator. Thus the induction process in the crucible and in the metal is almost unaffected by the presence of alumina. For safety reasons, the induction coil is also embedded in the alumina layer. Above this alumina layer there is a layer of another refractory material, called Plibrico. Moreover, for safety reasons there is a metal sheet surrounding the furnace, which prevents from high magnetic fields outside the furnace. The furnace rests on a base of concrete, which will be also considered in the computational domain. For the same purpose of thermal insulation, there is also placed a lid over the load, but it will not be considered it in the numerical simulation. Otherwise, we should solve an internal radiation problem, instead of imposing the convection-radiation boundary condition given in (25). In (Bermúdez et al., 2006; 2011) an improved thermal model including an internal radiation condition is introduced.

The computational domain for the electromagnetic problem consists of the furnace (load, crucible and coil) as it is shown in Fig. 7. The computational domain for the thermal problem is composed by the metal, the crucible, the refractory layers and the coil. The computational domain for the hydrodynamic problem is the molten metal and it is computed at each time step.

Concerning the values of the physical properties of the different materials, we show only the electrical conductivity of the crucible and metal to be melted because it plays a major role in the results. We notice that the electrical conductivity depends on temperature, being this dependency really important in the second case, because the load is assumed to be an insulator when solid and a good conductor when it melts, as can be seen in Fig. 8. 


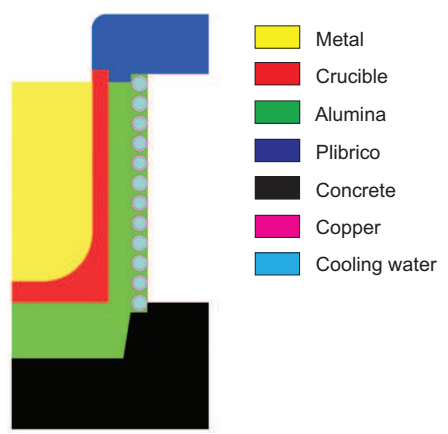

Fig. 7. Distribution of materials in the radial section of the furnace.

In the electromagnetic model that we have presented two working parameters have to be chosen: the frequency of the alternating current and the corresponding current intensity. However, when trying to simulate the real furnace, the given data is the power, and the current intensity is adjusted to obtain that power in the furnace. Moreover, since the electrical conductivity of the materials varies with temperature, the relationship between power and intensity also changes, and the intensity is dynamically adjusted during the process. To deal with this difficulty, the algorithm was slightly modified (see (Bermúdez et al., 2011) for further details) to provide the power as the known data and then to compute the intensity to attain the given power. Thus, for the numerical simulation, we have used the total power of the system as data.

\subsection{Numerical results}

We have performed two numerical simulations of the furnace with the same value of the power and two different values of the frequency, $500 \mathrm{~Hz}$ and $2650 \mathrm{~Hz}$, to see how the frequency affects the heating and stirring of the metal.

In Fig. 9 we represent the temperature in the furnace for each simulation. As it can be seen the temperatures obtained in the furnace are very similar, but a little higher in the case of the highest frequency. We notice the strong influence of the refrigeration tubes in the temperature: the temperature in the copper coil and the surrounding refractory is about $50{ }^{\circ} \mathrm{C}$, causing a very large temperature gradient within the refractory layer. In Fig. 10 we show a detail of the temperatures in the crucible and in the load. As it can be seen, higher temperatures are
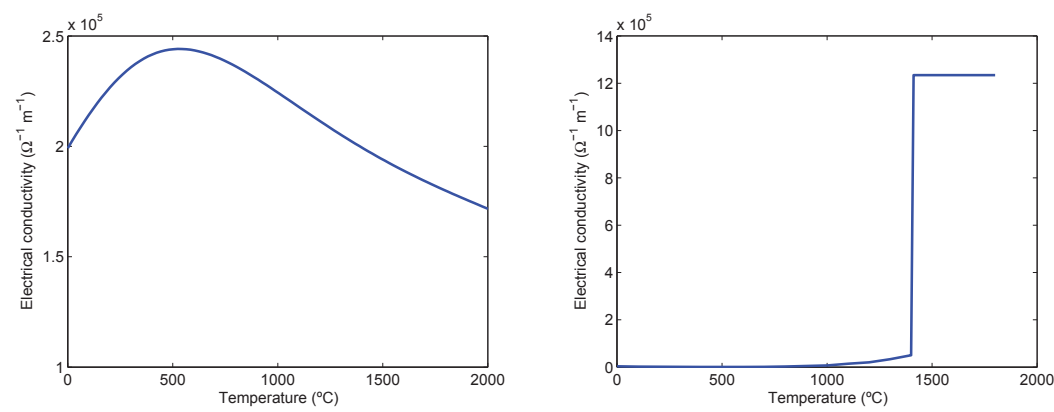

Fig. 8. Electrical conductivity for the crucible (left) and the load (right). 

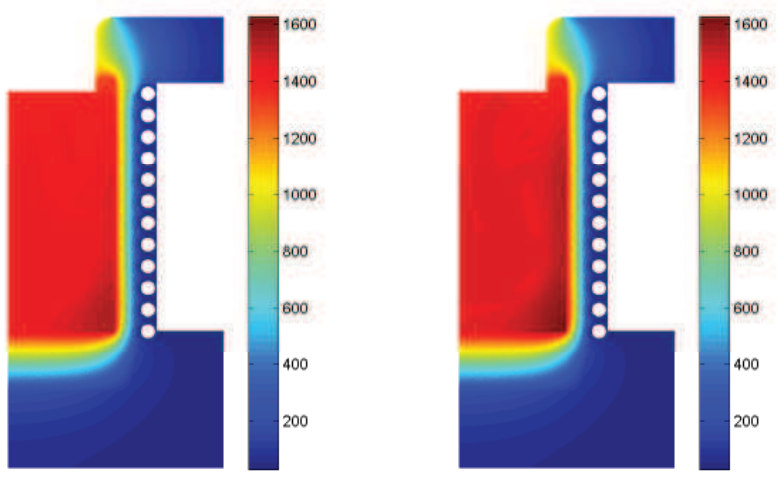

Fig. 9. Temperature after three hours for $500 \mathrm{~Hz}$ (left) and $2650 \mathrm{~Hz}$ (right).

reached when working with high frequency. This fact is explained due to the distribution of ohmic losses, as we will explain below.

In Fig. 11 we show the Joule effect in the load and the crucible after five minutes, when the metal is still solid. In Fig. 12, the same field is represented after three hours, when the metal has been melted. Comparing the results for different frequencies, we can see that the higher the frequency the higher the maximum values of the Joule effect, but due to the skin effect they are concentrated on the external wall of the crucible. Decreasing the frequency allows a better power distribution, at the cost of using higher intensities, thus causing larger power losses in the coil (see Fig. 14).

Moreover, comparing the results for solid and molten load, we see how the high conductivity of the molten metal affects the performance of the furnace. At low frequency the ohmic losses in the load become higher when the material melts, thus heating the load directly and reducing the crucible temperature (see Fig. 14 for the ohmic losses and Fig. 10 for the crucible temperature). Moreover, the presence of molten material increases the skin effect on
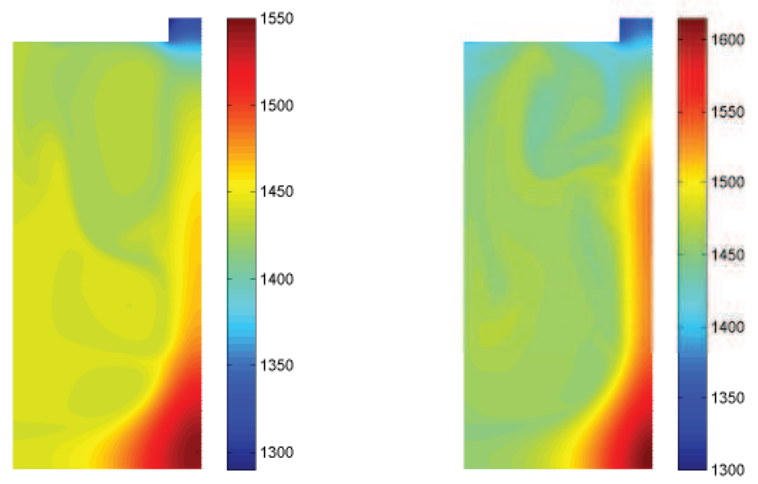

Fig. 10. Metal temperature after three hours for $500 \mathrm{~Hz}$ (left) and $2650 \mathrm{~Hz}$ (right). 

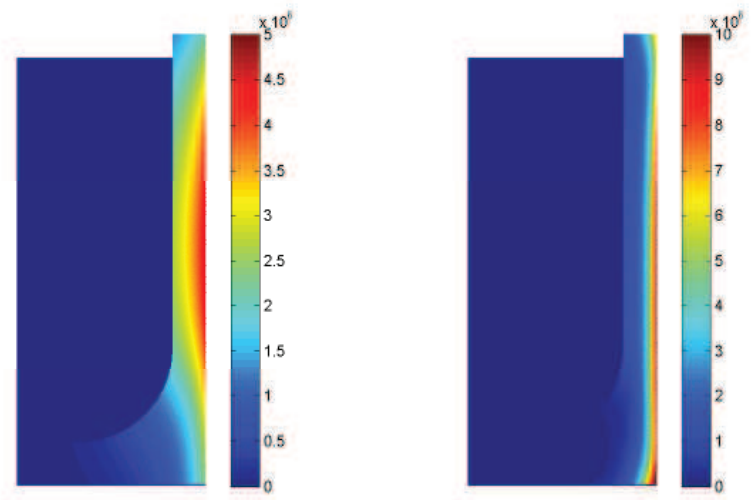

Fig. 11. Joule effect after five minutes for $500 \mathrm{~Hz}$ (left) and $2650 \mathrm{~Hz}$ (right).

the crucible wall. On the contrary, when working with high frequency the power distribution in the furnace remains almost unaffected in the presence of molten material.

We also present in Fig. 13 the velocity field for both frequencies. When working with low frequency the depth of penetration is higher, and Lorentz's force becomes stronger than buoyancy forces. At high frequency, instead, the low skin depth makes Lorentz's force almost negligible and buoyancy forces become dominant. This can be seen in this figure: at high frequency the molten metal is moving by natural convection, thus it tends to go up near the hot crucible, except in the upper part, probably due to the boundary condition we are imposing. At low frequency the electromagnetic stirring enforces the metal to go down close to the crucible, and a new eddy comes up in the bottom of the furnace.

Finally, in Fig. 14 we represent the variation in time of the Joule effect in each material, along with the heat losses through the refrigeration tubes. In order to attain the desired power, higher intensities are needed working at low frequency, which causes stronger ohmic losses in the copper coil. Moreover, at low frequency the load begins to melt after 60 minutes, affecting
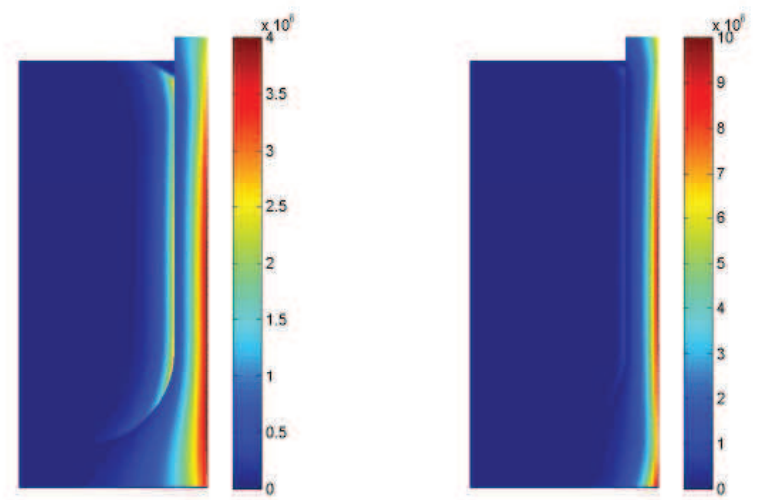

Fig. 12. Joule effect after three hours for $500 \mathrm{~Hz}$ (left) and $2650 \mathrm{~Hz}$ (right). 

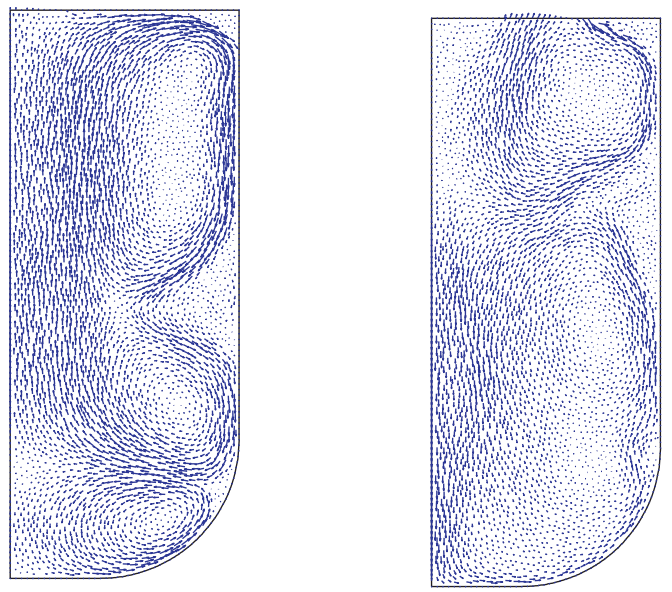

Fig. 13. Velocity fields after three hours for $500 \mathrm{~Hz}$ (left) and $2650 \mathrm{~Hz}$ (right).
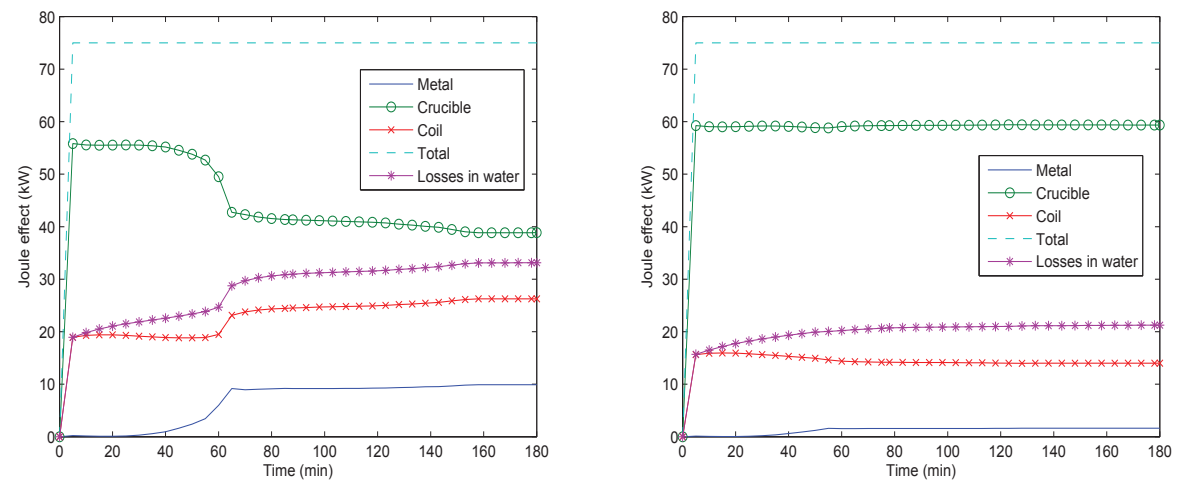

Fig. 14. Joule effect and heat losses through the tubes, for $500 \mathrm{~Hz}$ (left) and $2650 \mathrm{~Hz}$ (right).

the performance of the furnace and enforcing to increase the intensity, consequently increasing the power losses in the coil. When working at high frequency the performance of the furnace is almost unaffected by the presence of molten material. It is also remarkable that at the first time steps the power losses in the coil match the heat losses through the water tubes. When time increases the heat losses through the tubes become higher due to the heat conduction from the crucible across the refractory layer.

\section{Acknowledgements}

This work was supported by Ministerio de Ciencia e Innovación (MICINN), Spain, under research projects MTM2008-02483 and Consolider MATHEMATICA CSD2006-00032, by Xunta de Galicia, Spain, under grant number INCITE09-207047-PR (A. Bermúdez, D. Gómez and P. Salgado) and research project 10PXIB291088PR (M.C. Muñiz) and by the European Research Council through the FP7 Ideas Starting Grant 205004 (R. Vázquez). 


\section{References}

Alonso Rodríguez, A. M. \& Valli, A. (2008). Voltage and current excitation for time-harmonic eddy-current problems. SIAM J. Appl. Math., Vol. 68, 1477-1494.

Amrouche, C.; Bernardi, C.; Dauge, M. \& Girault, V. (1998). Vector potentials in three-dimensional non-smooth domains, Math. Meth. Appl. Sci., Vol. 21, 823-864.

Ammari, H.; Buffa, A. \& Nédélec, J.C. (2000). A justification of eddy currents model for the Maxwell equations, SIAM J. Appl. Math., Vol. 60, No. 5, 1805-1823.

Bay, F.; Labbe, V.; Favennec, Y. \& Chenot, J.L. (2003). A numerical model for induction heating processes coupling electromagnetism and thermomechanics, Int. J. Numer. Meth. Engng., Vol. 5, 839-867.

Bermúdez, A.; Moreno, C.; (1994). Duality methods for solving variational inequalities, Comput. Math. Appl., Vol. 7, 43-58.

Bermúdez, A.; Bullón, J.; Pena, F. \& Salgado, P. (2003). A numerical method for transient simulation of metallurgical compound electrodes, Finite Elem. Anal. Des., Vol. 39, 283-299.

Bermúdez, A.; Gómez, D.; Muñiz, M.C. \& Salgado, P. (2007). Transient numerical simulation of a thermoelectrical problem in cylindrical induction heating furnaces, Adv. Comput. Math.,Vol. 26, 39-62.

Bermúdez, A.; Gómez, D.; Muñiz, M.C. \& Salgado, P. (2007). A FEM/BEM for axisymmetric electromagnetic and thermal modelling of induction furnaces, Internat. J. Numer. Methods Engrg.,Vol. 71, No. 7, 856-882.

Bermúdez, A.; Gómez, D.; Muñiz, M.C. \& Salgado, P. \& Vázquez, R. (2009). Numerical simulation of a thermo-electromagneto-hydrodynamic problem in an induction heating furnace, Applied Numerical Mathematics, Vol. 59, No. 9, 2082-2104.

Bermúdez, A.; Gómez, D.; Muñiz, M.C. \& Vázquez, R. (2011). A thermoelectrical problem with a nonlocal radiation boundary condition, Mathematical and Computer Modelling, Vol. 53, 63-80.

Bermúdez, A.; Leira, R.; Muñiz, M.C. \& Pena, F. (2006). Numerical modelling of a transient conductive-radiactive thermal problem arising from silicon purification, Finite Elem. Anal. Des., Vol. 42, 809-820.

Bossavit, A. (1998). Computational electromagnetism, Academic Press Inc., San Diego, CA.

Brezzi, F. \& Fortin, M. (1991). Mixed and hybrid finite element methods, Springer Verlag, New York.

Chaboudez, C.; Clain, S.; Glardon, R.; Mari, D.; Rappaz, J. \& Swierkosz, M. (1997). Numerical Modeling in Induction Heating for Axisymmetric Geometries, IEEE Trans. Magn.,Vol. 33, 739-745.

Henneberger, G. \& Obrecht, R. (1994). Numerical calculation of the temperature distribution in the melt of industrial crucible furnaces, Second International Conference on Computation in Electromagnetics.

Hiptmair, R. \& Sterz, O. (2005). Current and Voltage Excitation for the Eddy Current Model, Int. J. Numer. Modelling, Vol. 18, No. 1, 1-21.

Hömberg, D. (2004). A mathematical model for induction hardening including mechanical effects, Nonlinear Anal. Real World Appl., Vol. 5, 55-90.

Katsumura, Y.; Hashizume, H. \& Toda, S. (1996). Numerical Analysis of fluid flow with free surface and phase change under electromagnetic force, IEEE Trans. Magn., Vol. 32, 1002-1005.

Lavers, J. (1983). Numerical solution methods for electroheat problems, IEEE Trans. on Magn., 
Vol. 19, 2566-2572.

Lavers, J. (2008). State of the art of numerical modelling for induction processes, COMPEL, Vol. 27, 335-349.

Mohammadi, B. \& Pironneau, O. (1994). Analysis of the k-epsilon turbulence model, Wiley/Masson, New York.

Natarajan, T.T. \& El-Kaddah, N. (1999). A methodology for two-dimensional finite element analysis of electromagnetically driven flow in induction stirring systems, IEEE Trans. Magn.,Vol. 35, No. 3, 1773-1776.

Nédélec, J.C. (2001). Acoustic and electromagnetic equations. Integral representations for harmonic problems, Springer-Verlag, New York.

Elliot, C. \& Ockendon, J.R. (1985). Weak and Variational Methods for Free Boundary Problems, Pitman, London.

Pironneau, O. (1982). On the transport-diffusion algorithm and its applications to the Navier-Stokes equations, Numer. Math., Vol. 38, No. 3, 309-332.

Rappaz, J. \& Swierkosz, M. (1996). Mathematical modelling and numerical simulation of induction heating processes, Appl. Math. Comput. Sci., Vol. 6, No. 2, 207-221.

Vázquez, R. (2008). Contributions to the mathematical study of some problems in magnetohydrodynamics and induction heating, PhD thesis, Universidade de Santiago de Compostela, Spain.

Wilcox, D.C. (1998). Turbulence modeling for CFD, DCW Industries, Inc. 


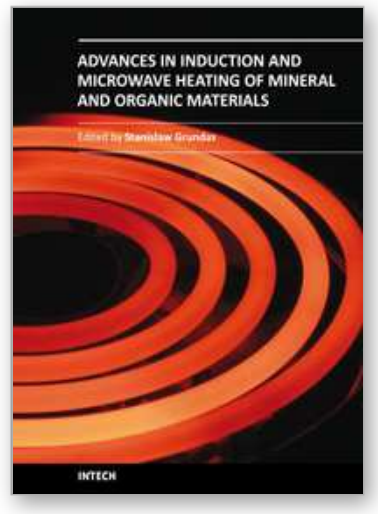

\author{
Advances in Induction and Microwave Heating of Mineral and \\ Organic Materials
}

Edited by Prof. StanisÅ,aw Grundas

ISBN 978-953-307-522-8

Hard cover, 752 pages

Publisher InTech

Published online 14, February, 2011

Published in print edition February, 2011

The book offers comprehensive coverage of the broad range of scientific knowledge in the fields of advances in induction and microwave heating of mineral and organic materials. Beginning with industry application in many areas of practical application to mineral materials and ending with raw materials of agriculture origin the authors, specialists in different scientific area, present their results in the two sections: Section 1-Induction and Microwave Heating of Mineral Materials, and Section 2-Microwave Heating of Organic Materials.

\title{
How to reference
}

In order to correctly reference this scholarly work, feel free to copy and paste the following:

A. Bermúdez, D. Gómez, M.C. Muñiz, P. Salgado and R. Vázquez (2011). Numerical Modelling of Industrial Induction, Advances in Induction and Microwave Heating of Mineral and Organic Materials, Prof. StanisÅ,aw Grundas (Ed.), ISBN: 978-953-307-522-8, InTech, Available from:

http://www.intechopen.com/books/advances-in-induction-and-microwave-heating-of-mineral-and-organicmaterials/numerical-modelling-of-industrial-induction

\section{INTECH}

open science | open minds

\section{InTech Europe}

University Campus STeP Ri

Slavka Krautzeka 83/A

51000 Rijeka, Croatia

Phone: +385 (51) 770447

Fax: +385 (51) 686166

www.intechopen.com

\section{InTech China}

Unit 405, Office Block, Hotel Equatorial Shanghai

No.65, Yan An Road (West), Shanghai, 200040, China

中国上海市延安西路65号上海国际贵都大饭店办公楼405单元

Phone: +86-21-62489820

Fax: $+86-21-62489821$ 
(C) 2011 The Author(s). Licensee IntechOpen. This chapter is distributed under the terms of the Creative Commons Attribution-NonCommercialShareAlike-3.0 License, which permits use, distribution and reproduction for non-commercial purposes, provided the original is properly cited and derivative works building on this content are distributed under the same license. 